\title{
Coordinated Buckling of Thick Multi-Walled Carbon Nanotubes Under Uniaxial Compression
}

\author{
Xu Huang ${ }^{1}$, Hongyan Yuan ${ }^{1}$, K. Jimmy Hsia ${ }^{2}$, and Sulin Zhang ${ }^{1}(\varangle)$ \\ ${ }^{1}$ Department of Engineering Science and Mechanics, The Pennsylvania State University, University Park, PA 16802, USA \\ ${ }^{2}$ Department of Mechanical Science and Engineering, University of Illinois, Urbana, IL 61801, USA \\ Received: 15 October 2009 / Revised: 17 November 2009 / Accepted: 18 November 2009 \\ (C) The Author(s) 2010. This article is published with open access at Springerlink.com
}

\begin{abstract}
Using a generalized quasi-continuum method, we characterize the post-buckling morphologies and energetics of thick multi-walled carbon nanotubes (MWCNTs) under uniaxial compression. Our simulations identify for the first time evolving post-buckling morphologies, ranging from asymmetric periodic rippling to a helical diamond pattern. We attribute the evolving morphologies to the coordinated buckling of the constituent shells. The post-buckling morphologies result in significantly reduced effective moduli that are strongly dependent on the aspect ratio. Our simulation results provide fundamental principles to guide the future design of high-performance, MWCNT-based nanodevices.
\end{abstract}

\section{KEYWORDS}

Carbon nanotubes, buckling, uniaxial compression, rippling

\section{Introduction}

Multi-walled carbon nanotubes (MWCNTs) have found a wide range of applications as basic building blocks in micro(nano)-electromechanical systems (MEMS/NEMS) [1-5], owing to their unique combination of mechanical, electronic, and biochemical properties. Since carbon nanotubes (CNTs) are slender, thin-walled structures that are susceptible to buckling under compressive loading, failure induced by mechanical instability has been a major concern in the performance of such CNT-based nanodevices [5]. The behavior of single-walled CNTs (SWCNTs) under different loading conditions (bending, twisting, and compression) has been well characterized by various methods, ranging from all-atom molecular dynamics (MD) simulations [6-10] to continuum beam $[8,11]$ and shell theory-based modeling $[8,12]$. These studies predicted that the buckling morphologies feature a single local kink in SWCNTs beyond a critical bending strain. This phenomenon highlights the very large energetic cost of stretching a CNT compared to bending it. In bent and twisted MWCNTs, the single sharp kink is replaced by periodic undulations [5, 12-22]. Specifically, the Yoshimura (diamond shaped) pattern appears in the compressive section of bent MWCNTs [5, 13-15, 17, 18, 21, 23], whilst periodic wave-like ripples develop in both the length and circumferential directions in twisted MWCNTs $[13,14,16,22,23]$. These deformation morphologies result in a substantially reduced effective modulus $[13,14,22-26]$. The mechanical responses of MWCNTs

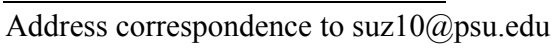


are generally reversible upon unloading, indicating their nonlinear, purely elastic nature.

An MWCNT is composed of a number of concentric thin shells. The aspect ratios (tube length divided by tube diameter) of the innermost and outermost shells of a given MWCNT may differ by two orders of magnitude. Under uniaxial compression, isolated shells with the innermost shell aspect ratio may exhibit beam (columnar) buckling, while those with the outermost shell aspect ratio exhibit shell buckling. In reality, the inter-shell van der Waals (vdW) interactions play a critical role, constraining the buckling of these constituent shells. It is thus interesting to examine which buckling mode an MWCNT will adopt under the influence of inter-shell vdW interactions.

Experimental evidence of the instability of uniaxially compressed MWCNTs has recently emerged [27-29]. Waters et al. [27, 28] conducted nanoindentation measurements of vertically aligned short, thick MWCNT forests (with aspect ratio ranging from 1 to 2). Hysteresis was observed in their load-displacement curve, which indicates the occurrence of plastic deformation with two possible sources: defect nucleation (Stone-Wales defects) and propagation [30-35], or adhesions between neighboring MWCNTs under the compressive loading. Using scanning electron microscopy (SEM), they found that under compression the buckled MWCNTs exhibited strongly distorted cross-sections and wrinkled sidewalls. Due to the large size of the nanoindentation tips used in their experiments, several MWCNTs were simultaneously compressed. At a certain stage of the indentation, neighboring MWCNTs may come to contact with each other, which poses difficulties in the post-buckling analysis for a single MWCNT. They also found that the measured buckling strain is neither consistent with the prediction by continuum theory using the elasticity parameters of Yakobson et al. [8], nor with the theoretical model developed by $\mathrm{Ru}$ [36]. Using atomic force microscopy (AFM), Yap et al. [26] characterized the instability of individual MWCNTs with different aspect ratios under cyclic compression. While their experiments unambiguously demonstrated that instability of MWCNTs with large aspect ratios is governed by Euler buckling, the post-buckling deformation morphology of MWCNTs with relatively small aspect ratios remains unknown, largely due to the experimental difficulties in in situ imaging of the deformed MWCNTs with nanoscale resolutions. Yap et al. hypothesized that the instability of short, thick MWCNTs can be attributed to symmetric shell buckling.

All-atom simulations with empirical interatomic potentials have been widely used to study the deformation of CNTs. However, for the study of thick MWCNTs, fully atomistic simulations are computationally prohibitive because of the large number of degrees of freedom involved. This explains why most previous atomistic studies have been limited to SWCNTs or MWCNTs with only a few layers. In this article, we systematically characterize the deformation morphologies and energetics of thick MWCNTs $(\geqslant 10$ layers) with relatively small aspect ratio under uniaxial compression through a large-scale, effective quasi-continuum method, and rationalize the buckling mechanisms. Our simulations identify evolving postbuckling morphologies, ranging from asymmetric periodic rippling to helical diamond patterns. We attribute the evolving morphologies to the coordinated buckling of the constituent shells. We further note that the post-buckling morphologies result in significantly reduced effective moduli that are strongly dependent on the aspect ratio. Our simulation results may provide fundamental principles to guide the design of future high-performance, MWCNT-based nanodevices.

\section{Methodology}

We adopt the modified second-generation Brenner potential [37], denoted by MTB-G2, to describe the short-range covalent interactions in MWCNTs; this takes the form:

$$
V_{\mathrm{TB}}=\sum_{i} \sum_{j>i}\left[V^{\mathrm{R}}\left(r_{i j}\right)-B_{i j}(r) V^{\mathrm{A}}\left(r_{i j}\right)\right]
$$

where $r_{i j}$ is the distance between atoms $i$ and $j, V^{\mathrm{R}}$ and $V^{\mathrm{A}}$ are the pairwise repulsive and attractive interactions, respectively, and $B_{i j}$ is the bond-order function that has a complicated dependence on the bond angles and bond lengths involving atoms $i$ and $j$. The long-range nonbonding interaction is described 
by a Lennard-Jones (LJ) potential with the parameters given by Girifalco et al. [38], as

$$
V_{\mathrm{LJ}}(r)=\frac{\epsilon}{r_{0}^{6}}\left[\frac{1}{2} \kappa^{6}\left(\frac{r_{0}}{r}\right)^{12}-\left(\frac{r_{0}}{r}\right)^{6}\right]
$$

where $r$ is an interatomic distance, $\kappa=2.7$ is a dimensionless constant, $r_{0}=1.42 \AA$ is the equilibrium bond length, and $\epsilon=15.2 \mathrm{eV} \cdot \AA^{6}$.

To improve the computational affordability without sacrificing accuracy, the fully atomistic models employed are here coarse-grained by a quasicontinuum method based on the finite crystal elasticity theory for curved crystalline monolayers [39-41]. Within this theoretical framework, the exponential Cauchy-Born rule was proposed as a way of linking the kinematics at the atomic and continuum scales:

$$
a=\zeta(A)
$$

where $\zeta$ is an exponential map that transforms the undeformed lattice vector $\boldsymbol{A}$ into a deformed one $\boldsymbol{a}$. Through a local approximation of the exponential map, the deformed lattice vectors and the angles between two lattice vectors can be analytically represented in terms of the continuum deformation measures of the surface. Consider a representative unit cell of area $S_{0}$ containing two inequivalent nuclei and three inequivalent bonds in the reference, undeformed configuration: the hyperelastic strain energy density $W$ can be formulated in terms of the MTB-G2 potential,

$$
\begin{aligned}
W & =W(\boldsymbol{C} ; \boldsymbol{K} ; \boldsymbol{\eta}) \\
& =\frac{1}{S_{0}} \sum_{i=1}^{3}\left[V^{\mathrm{R}}\left(\boldsymbol{a}_{i}\right)+b\left(\boldsymbol{a}_{i}, \boldsymbol{a}_{j}, \boldsymbol{a}_{k}, \theta_{j}, \theta_{k}\right) V^{\mathrm{A}}\left(\boldsymbol{a}_{i}\right)\right]
\end{aligned}
$$

where $C$ and $K$ are the stretch and curvature tensors, respectively; $\boldsymbol{a}_{i}, \boldsymbol{a}_{j}, \boldsymbol{a}_{k}$ are the three inequivalent bonds, $\theta_{j}, \theta_{k}$ are the angles between these three bonds, and $S_{0}=(3 \sqrt{3} / 2)\|A\|^{2}$ is the area of the unit cell. Note that the hexagonal lattice is a Bravais multi-lattice, so an additional kinematic variable $\eta$ must be introduced to describe the relative shift between the two basic lattices. This inner variable is eliminated by minimizing the strain-energy density function, giving rise to a stable local lattice arrangement within the unit cell.
After this inner relaxation, the strain energy density can be written as a function of only $C$ and $K$ :

$$
\hat{W}=\hat{W}[C ; K ; \eta(C ; K)]
$$

Thus, the continuum representation of the covalent binding energy for the shells in an MWCNT subject to the deformation map $\phi$ that maps from the undeformed to deformed configurations is

$$
E=\sum_{i=1}^{n} \int_{\Omega_{i}} \hat{W}[C[\phi(X)] ; K[\phi(X)]] \mathrm{d} \Omega_{i}
$$

where $X$ is a material point in the undeformed configuration, and $\Omega_{i}$ is the surface area of the $i$-th shell in an $n$-walled MWCNT.

Homogenization of the discrete nonbonded energy density between two unit cells gives rise to the $\mathrm{vdW}$ energy density, as

$$
V_{\text {vdW }}(r)=\left(\frac{2}{S_{0}}\right)^{2} V_{\mathrm{LJ}}(r)
$$

The factor of two on the right-hand side of Eq. (7) comes from the fact that each unit cell contains two nuclei. The nonbonded energy between two neighboring shells is then

$$
E_{\mathrm{vdW}}=\frac{1}{2} \sum_{i=1}^{n-1} \int_{\Omega_{0}^{i}} \int_{\Omega_{0}^{i+1}} V_{\mathrm{vdW}}\left[\left\|\phi\left(\boldsymbol{X}_{i}\right)-\phi\left(\boldsymbol{X}_{i+1}\right)\right\|\right] \mathrm{d} \Omega_{0}^{i} \mathrm{~d} \Omega_{0}^{i+1}
$$

where $X_{\mathrm{i}}$ and $X_{\mathrm{i}+1}$ are the two material points that are on the $i$-th and (i+1)-th shells, respectively, in the MWCNT; $\Omega_{0}^{i}$ and $\Omega_{0}^{i+1}$ are the surfaces of the $i$-th and (i+1)-th shells, respectively.

Based on the coarse-grained constitutive relations for both the bonding and nonbonding interactions, the constituent shells of the MWCNTs are discretized by finite elements. In our simulations, an $n$-walled MWCNT is indexed by $(5,5) /(10,10) / \ldots /(5 n, 5 n)$, where the inter-shell spacing is roughly $0.34 \mathrm{~nm}$. As the first step, the MWCNTs are fully relaxed free of any constraints using a limited-memory BroydenFletcher-Goldfarb-Shanno (BFGS) algorithm [42]. A ring of finite-element nodes at each end of the MWCNTs are then held fixed and the MWCNTs are 
uniaxially compressed by stepwise movement of the fixed nodes to the center in the axial direction. At each step, the MWCNTs are relaxed under the prescribed compressive strain; the reaction forces at the ends and the deformation morphology can be extracted from the equilibrated configurations.

The coarse-grained model reduces the number of degrees of freedom by two orders of magnitude as compared to its atomistic counterpart, yet remains faithful to the nonlinearity of the interatomic interactions and the basic characteristics of hexagonal lattices. For instance, the coarse-grained model inherits the chirality of the atomistic counterpart, and gives rise to the correct stacking energy between the neighboring shells. Thus, the coarse-grained model reproduces the deformation morphologies and energetics of the corresponding atomistic models for defect-free, homogeneously deformed CNTs.

\section{Results and discussion}

\subsection{Evolving post-buckling morphologies}

We choose a 20-walled CNT as a representative example to illustrate the deformation morphologies and energetics of thick MWCNTs under uniaxial compression. The aspect ratio of the MWCNT is $\rho=L / d=5$, where $L$ is the length, and $d$ is the diameter of the outermost wall of the MWCNT (also regarded as the diameter of the MWCNT). Figure 1 shows the deformation morphologies at different compressive strains. The deformation morphologies of four constituent shells-the innermost, the 5th, the 10th, and the outermost shells-are shown from left to right in each of Figs. 1(a)-1(c). We observed that, upon constraint-free geometry optimization as the first step (Fig. 1(a)), the shells in the MWCNT undergo rigid rotation with respect to their central axis. From innermost to outermost layers, the neighboring shells rotate in alternating directions (clockwise and counter clockwise). We attribute this alternating rotation to the registry-dependent inter-shell interactions [43]. In particular, the arrangement of the neighboring shells in the initial configuration (the initial guess) is close to A-A stacking, which is energetically disfavored [44]. The rotations of the neighboring shells in alternating directions rearrange the neighboring shells from $\mathrm{A}-\mathrm{A}$ to A-B stacking, which lowers the inter-shell vdW interactions without affecting the in-plane deformation energetics. This rearrangement shows the capability of the coarse-grained model to inherit the chirality of its atomistic counterpart.

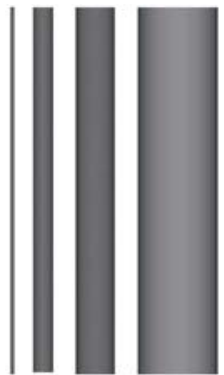

(a)

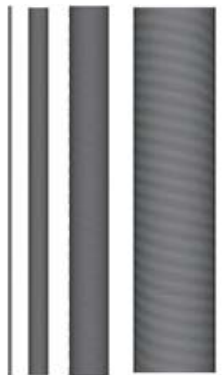

(b)

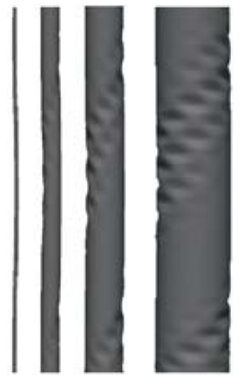

(c)
Figure 1 Evolution of the deformation morphologies of a uniaxially compressed 20-walled MWCNT. Each subfigure consists of four constituent shells of the 20-walled MWCNT. From left to right in each subfigure, the shells are the innermost, the 5th, the 10th, and the outermost shells. (a) Fully relaxed, undeformed configuration. (b) At a compressive strain of $\varepsilon=8.23 \times 10^{-3}$, wave-like ripples appear along the 10 th and the outermost shells, while ripples are not observable in the other two inner shells. (c) At a compressive strain of $\varepsilon=1.18 \times 10^{-2}$, a helical diamond pattern runs from one end to the other for the outer three shells. The innermost shell is further bent and twisted throughout its length

As the compressive strain increases, all shells of the MWCNT undergo uniform shortening. At a critical compressive strain of $\varepsilon=8.23 \times 10^{-3}$, wave-like periodic ripples appear (Fig. 1(b)) along the tube. The rippling pattern, however, is graphically undetectable in the two innermost shells. Such a rippling morphology in the inner shells would cause a large deformation energy penalty since inner shells are much more rigid. The rippling amplitude progressively increases from inner to outer shells, and increases with increasing compressive strain. The rippling in all shells has the same rippling periodicity, manifesting the coordinated nature of the deformation of the constituent shells. It is interesting to note that the rippling pattern is not exactly perpendicular to the axial direction (the loading direction) of the MWCNT, but has a small tilt. With a slightly increased compressive strain, the rippling pattern evolves into a diamond buckling pattern (Fig. 1(c)). Figure 1(c) shows 
that at $\varepsilon=1.18 \times 10^{-2}$ a helical diamond pattern appears in the three outer shells. In contrast, the innermost shell deviates from its linear configuration, but without a graphically visible diamond pattern. The helical diamond patterns predicted by our simulations have not been observed in either other numerical simulations or previous experiments.

The constrained deformation of the innermost tube merits detailed study. Figure 2 displays the deformation mode of the innermost tube in the 20-walled MWCNT at different applied strains. To aid visualization of the deformed configurations (in green), a reference tube (in blue) is also plotted. The reference tube represents the uniformly shortened configuration without any radial displacement under the applied strain. The radial displacements of the deformed tubes due to uniaxial compression are rescaled by a factor of 20 to further aid visualization. At a compressive strain of $\varepsilon=8.23 \times 10^{-3}$ at which wave-like ripples appear (Fig. 1(b)), the innermost tube remains straight (Fig. 2(a)) so that the deformed and the reference tubes overlap. At the critical strain of $\varepsilon=1.18 \times 10^{-2}$, the innermost tube is deformed into a spiral configuration around the reference tube (Fig. 2(b)). At the same critical strain, the helical diamond pattern on the outer shells also appears, indicating a coordinated buckling mechanism of the inner and outer shells. A further increase in the applied compressive strain results in an increase in the spiral amplitude (Figs. 2(c) and 2(d)). The innermost tube is simultaneously bent and twisted, as seen from the twist marks in the spiral configurations and confirmed by the calculated reaction forces at the ends of the tube.

\subsection{Deformation energetics}

To further reveal the physical mechanisms governing the evolution of the deformation morphologies, we plot in Fig. 3(a) the total system energy (in units of aJ) and the inter-shell nonbonding energy (vdW interaction energy) as a function of the applied compressive strain. The fully relaxed, undeformed configuration is taken to be the reference energy state. For comparison, the energy of the MWCNT undergoing an idealized deformation is also plotted, where the deformation of the MWCNT is perfectly cylindrical without rippling or diamond pattern formation throughout the entire loading process. The two sets of curves are graphically indistinguishable up to the bifurcation point and thereafter deviate gradually from each other. This bifurcation point corresponds to the onset of ripple formation in the MWCNT. In the pre-bifurcation regime, the total system energy increases quadratically with respect to the compressive strain, while the inter-shell nonbonding energy decreases linearly due to the uniform shortening of the MWCNT upon compression. In the post-bifurcation regime, the inter-shell nonbonding energy stored in the buckled morphology is much higher than in the idealized case, while the total system potential energy is still lower. This clearly suggests that the release of in-plane strain energy acts as the driving force for the rippling and diamond pattern formation, and is opposed by the increase in the inter-shell nonbonding energy. Since the in-plane stiffness in MWCNTs is much higher than the transverse stiffness, the rippling deformation and diamond pattern effectively release the in-plane strain energy, leading to an energetically favorable state at sufficiently high compressive load.

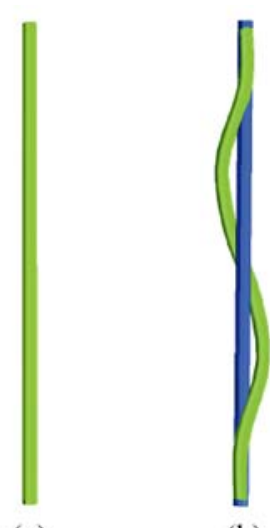

(a) (b)

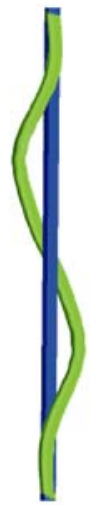

(c)

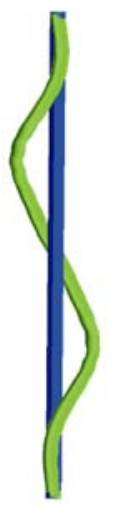

(d)
Figure 2 The deformation configurations of the innermost tube in the 20-walled MWCNT. The tube in blue represents the uniformly shortened configuration without any radial displacement under the applied compressive strains, while the tube in green represents the deformed configuration. To aid visualization, the radial displacements of the deformed tubes due to uniaxial compression are rescaled by a factor of 20 . At zero compressive strain, the configurations of these two tubes overlap. At finite applied strain, the deformed tube adopts a spiral configuration. The amplitude of the spiral configuration increases with increasing compressive strain. (a) $\varepsilon=8.23 \times 10^{-3}$; (b) $\varepsilon=1.18 \times 10^{-2}$; (c) $\varepsilon=1.29 \times 10^{-2}$; (d) $\varepsilon=1.47 \times 10^{-2}$ 


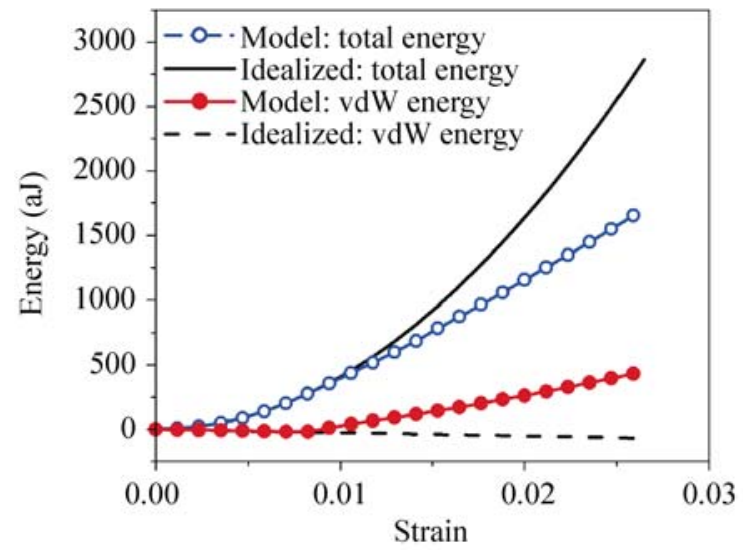

(a)

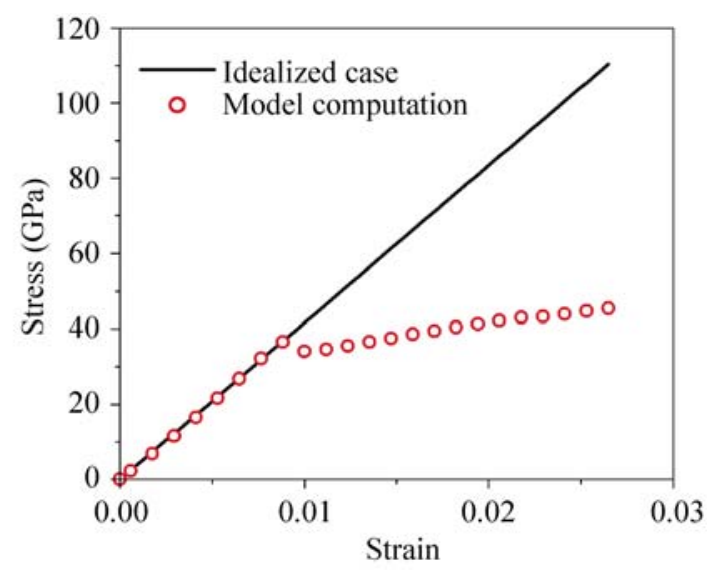

(b)

Figure 3 Energetics and mechanical responses of a 20-walled MWCNT under uniaxial compression. For comparison, an idealized case is also depicted, where the deformation of the MWCNT is constrained to a perfect cylindrical shape without buckling throughout the entire loading process. (a) As compared with the idealized deformation mode, the rippling deformation and diamond pattern formation beyond the buckling point releases the in-plane strain energy, penalized by the increase of the interlayer van der Waals (vdW) energy. The undeformed configuration is taken as the reference energy state. In the idealized case, the interlayer vdW energy decreases linearly due to the uniform shortening of the MWCNT upon compression. (b) Stress-strain relations. The post-buckling morphologies result in a substantially reduced effective modulus as compared to the pre-buckling regime

Figure 3(b) shows the stress-strain response of the MWCNT. For the idealized case, the compressive stress increases linearly with compressive strain throughout the entire loading process (solid black line). For the minimum energy path (red open circles) where the rippling and diamond pattern morphologies develop, the compressive stress first increases linearly with respect to the strain until a sudden drop appears, indicating the onset of local buckling. Beyond the buckling point, the compressive stress continues to increase with the applied strain, but with a significantly reduced stiffness as compared with that of the prebuckling regime. If the inter-shell vdW interaction energy is neglected, an MWCNT in the pre-buckling regime can be treated as a series of linear springs, with the effective modulus computed by

$$
Y=\frac{F}{\varepsilon\left(\pi t \sum_{i=1}^{n} d_{i}\right)}
$$

where $F$ is calculated reaction force at the applied compressive strain $\varepsilon$. The term in parenthesis in Eq. (9) is the effective cross-sectional area of the MWCNT, where $d_{i}$ is the radius of the $i$-th shell, and $t$ is the thickness of the shells. For the MTB-G2 potential, the Young's modulus of the shells is $\sim 243 / t \mathrm{~J} / \mathrm{m}^{2}$ based on the exponential Cauchy-Born rule [40] and $t=0.066 \mathrm{~nm}$ [8]. With these geometrical parameters, the calculated modulus is $251 / t \mathrm{~J} / \mathrm{m}^{2}$, which is fairly close to the analytical result (the difference is $\sim 3 \%$ ).

\subsection{Buckling mechanisms and onset buckling strain}

The buckling energetics and morphologies of a 20-walled MWCNT provide insights into the buckling mechanisms and the post-buckling responses of MWCNTs. To relate the buckling mechanisms of an MWCNT to that of its constituent shells, we note that the buckling mode of each constituent shell in the MWCNT depends on its aspect ratio. Buckling of an SWCNT with large aspect ratio follows the Euler beam theory, where the buckling strain is given by

$$
\varepsilon_{\mathrm{cr}}^{\text {beam }}=\frac{1}{2}(\pi / \rho)^{2}
$$

In contrast, SWCNTs with a small aspect ratio undergo shell buckling under compression with a buckling strain given by

$$
\varepsilon_{\mathrm{cr}}^{\text {shell }}=\frac{2 t}{d \sqrt{3\left(1-v^{2}\right)}}
$$

where $v$ is Poisson's ratio, and $v=0.397$ for the 
MTB-G2 potential [40]. Note that the buckling strain for shell buckling is independent of shell length.

For the $n$-walled MWCNT with an aspect ratio of $\rho$, the aspect ratio of the outermost shell is $\rho$, while that of the innermost shell is $n \rho$ under the condition that the innermost tube is $(5,5)$. Thus, for sufficiently large $n$, the innermost shell is prone to beam buckling, while the outermost shell undergoes shell buckling. The inter-shell nonbonding interaction has a constraining effect on both buckling modes, which delays the buckling as compared with isolated shells. For MWCNTs with relatively small aspect ratios (which are the focus of this study), it is conceivable that for the most general case there exist three critical buckling strains: the onset buckling strain for periodic rippling of the outermost shell $\varepsilon_{\text {rippling; }}^{\text {outer }}$ the onset buckling strain of the symmetric diamond pattern $\varepsilon_{\text {sym }}^{\text {outer }}$, and the onset buckling strain of the spiral configuration of the innermost shell $\varepsilon_{\text {spiral }}^{\text {inner }}$. Analytical evaluations of these critical strains require consideration of the inter-shell interactions, which is straightforward but lengthy. Depending on the aspect ratio and the thickness of the MWCNT, the range of the strain for symmetric buckling may be too narrow to be detectable. For example, in our simulations of 20-walled MWCNTs, the symmetric diamond pattern was not observed during the compression, indicating that $\varepsilon_{\text {sym }}^{\text {outer }}$ is fairly close to $\varepsilon_{\text {spiral }}^{\text {inner }}$. In contrast, for the 25-walled MWCNTs, as will be discussed later, there is a detectable strain range within which the symmetric diamond pattern appears. Since the focus of the present study is on the thick, short MWCNTs, the outermost shell tends to ripple first as the compressive strain reaches $\varepsilon_{\text {rippling }}^{\text {outer }}$. Due to the inter-shell vdW interactions, the rippling morphology propagates from the outermost shell all the way to the innermost shells. As the applied compressive strain increases, the rippling amplitude also increases. As the compressive strain reaches $\varepsilon_{\text {sym }}^{\text {outer }}$, rippling may no longer be the energetically favorable deformation mode, and may be replaced by the symmetric diamond pattern. Since the innermost shell is significantly more rigid in the transverse direction than the outermost shell, neither the ripple nor the diamond pattern is the energetically favorable deformation mode for the innermost shell. Accommodating the deformation mode propagated from the outermost shell thus results in significant strain energy in the innermost shell. To release this excessive strain energy, the innermost shell abandons the deformation mode propagated from the outermost shell, and itself bends. Due to the constraining effects of the outer shells, the deformation mode of the innermost shell adopts a spiral configuration. Such a spiral configuration of the innermost shell in turn propagates all the way to the outermost shell. In accommodating this deformation mode, the symmetric diamond pattern is disrupted, and replaced by the helical diamond pattern. The helical diamond pattern represents a hybrid buckling mode of the MWCNT when beam buckling of the innermost shell and shell buckling of the outermost shell are both activated. The buckling response of the MWCNT appears to be a result of the coordinated deformation of the innermost and outermost shells.

The periodic rippling is a characteristic shell buckling mode that has been widely observed in compressed thin-shell-like structures. Yet the tilting of the rippling demands further explanation. The tilted rippling pattern observed in the compressed MWCNTs resembles that observed in a twisted MWCNT $[13,14,16,22]$, which indicates that the MWCNT is internally twisted. The difference between these two rippling patterns is that the tilt angle of ripples in a twisted MWCNT is much larger. Examination of the reaction force at the tube ends reveals that the shells are indeed twisted, which explains the tilting of the rippling pattern. Since the applied strain is purely compressive, the torsion must be generated by internal relaxation of the shells. The torsion, and hence the tilting of the rippling pattern, may be attributed to the inter-shell lattice registry. The rippling pattern leads to an increase in surface area, which introduces an effective lattice mismatch of the neighboring shells. The lattice mismatch disrupts the originally established in-registry positions of the neighboring shells, raising the stacking energy between the shells. As a result, an inter-shell force (torsion in this case) is generated in order to return out-of-registry atoms to in-registry positions. The 
internally generated torsion lowers the total system energy, generating and locking the twist. A similar inter-shell registry driven twist has been observed in all-atom molecular mechanics simulations of bent MWCNTs [45].

We have systematically computed the buckling strains of a series of 1-, 2-, 5-, 10-, 15-, 20-, and 25-walled CNTs. The lengths of all these CNTs were the same, $L=68 \mathrm{~nm}$. Thus, their aspect ratios were different, ranging from 4 to 100. In Table 1 the numerically determined buckling strains are compared with the Euler beam theory (Eq. (10)) and the shell buckling theory (Eq. (11)). We found that these two theories describe well the extreme cases. The buckling strain of the SWCNT $((5,5)$ tube) agrees with the prediction of the Euler beam theory, while those of the 15-, 20-, and 25-walled CNTs show negligible differences from the predictions of the shell buckling theory. Note that in applying the shell buckling theory, a delay factor is added to Eq. (11) to take into account the stiffening effect stemming from the inter-shell nonbonding interactions. Specifically, the predicted buckling strain is $\alpha \varepsilon_{\text {cr }}^{\text {shell }}$, where $\alpha=1.41$. For moderate aspect ratios, however, both theories fail to predict the correct buckling strain.

Table 1 Comparisons of the computed buckling strains with the predictions by the Euler beam theory and the shell buckling theory. The lengths of all the CNTs considered here were the same, $L=68 \mathrm{~nm}$. For the SWCNT $(n=1)$, the computed buckling strain agrees well with that predicted by the Euler beam theory. For the 15-, 20-, and 25-walled MWCNTs, the computed buckling strains agree well with those predicted by the shell buckling theory. For moderate aspect ratios $(n=2-10)$, both theories fail to predict the buckling strains

\begin{tabular}{cccccc}
\hline & & & & \multicolumn{2}{c}{$\varepsilon$} \\
\cline { 5 - 6 }$n$ & $t / d$ & $d / L$ & $\varepsilon_{\text {cr }}$ & $\begin{array}{c}\text { Shell buckling } \\
\text { theory }\end{array}$ & $\begin{array}{c}\text { Euler beam } \\
\text { theory }\end{array}$ \\
\hline 1 & 0.0971 & 0.01 & 0.000493 & 0.164707 & 0.000493 \\
2 & 0.0485 & 0.02 & 0.002353 & 0.082353 & 0.001972 \\
5 & 0.0194 & 0.05 & 0.008230 & 0.032941 & 0.012325 \\
10 & 0.0097 & 0.10 & 0.013800 & 0.016471 & 0.049298 \\
15 & 0.0065 & 0.15 & 0.010580 & 0.010980 & 0.110921 \\
20 & 0.0049 & 0.20 & 0.008230 & 0.008235 & 0.197192 \\
25 & 0.0039 & 0.25 & 0.006568 & 0.006588 & 0.308113 \\
\hline
\end{tabular}

\subsection{Dependence of the aspect ratio on the post- buckling modulus}

Previous studies showed that the post-buckling responses of bent and twisted thick MWCNTs follow unique scaling laws $[13,14,16,22]$. To examine whether similar scaling laws exist for uniaxially compressed MWCNTs, we have systematically computed the stress-strain relations for a series of thick MWCNTs, including 10-, 15-, 20-, and 25-walled MWCNTs. Again, the length of these MWCNTs was fixed at $68 \mathrm{~nm}$, while the aspect ratios varied. The stressstrain relations for these MWCNTs are plotted in Fig. 4. In the pre-buckling regime, all the MWCNTs follow the same stress-strain relation, governed by the linear elasticity of the MWCNTs. Then, the compressive stress drops abruptly, indicating the onset of buckling. The buckling strain decreases with increasing thickness of the MWCNTs (and thus with decreasing aspect ratio). For all the MWCNTs, the post-buckling effective moduli are substantially smaller than that of the linear elastic regime. In addition, the post-buckling effective modulus increases monotonically with increasing thickness of the MWCNTs. For the 10-walled MWCNT, the effective post-buckling modulus is negative. Besides the helical

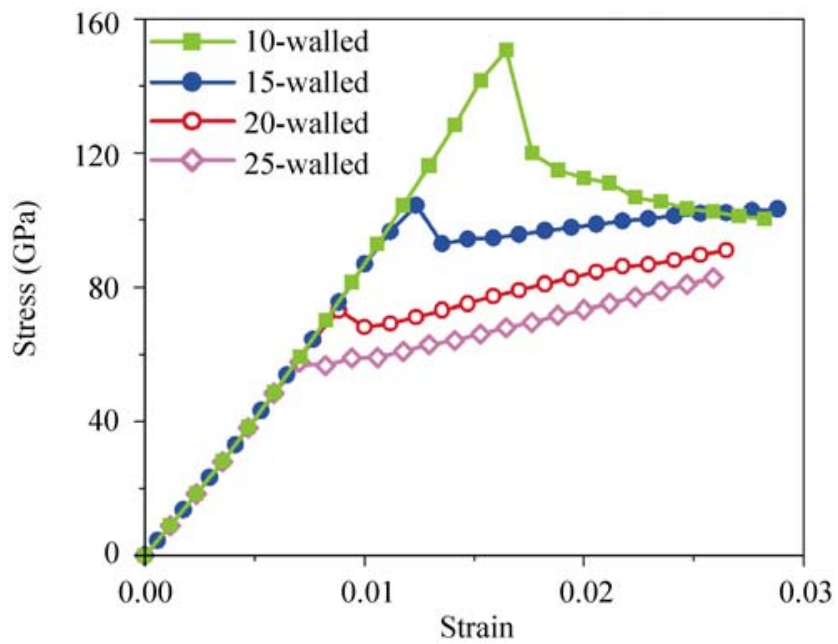

Figure 4 Stress-strain relations of thick MWCNTs under uniaxial compression. All the MWCNTs share the same modulus in the pre-buckling regime. The 10 -walled MWCNT has a negative post-buckling modulus, which is distinctly different from other MWCNTs due to the difference in the buckling mechanisms. The post-buckling response of 20- and 25-walled MWCNTs can be approximated by a linear law 
diamond pattern in the post-buckling regime, overall the 10-walled MWCNT is clearly bent, which is different from the other, thicker MWCNTs. This hybrid buckling mode explains why the buckling strain of the 10-walled CNT can neither be predicted by the Euler beam theory nor the shell buckling theory. In contrast, we did not observe global bending in the 15-, 20-, and 25-walled MWCNTs in their post-buckling morphologies within the range of the applied strain. Thus, their post-buckling mechanisms can be considered to be similar, as manifested by that fact that their buckling strains can all be described by shell buckling theory. Though the post-buckling stress-strain relations are intrinsically nonlinear, we note that for MWCNTs with smaller aspect ratios (the 20- and 25-walled MWCNTs), their post-buckling stress-strain relations are approximately linear over a wide range of the applied compressive strain, giving rise to nearly constant post-buckling moduli. This suggests that for thick MWCNTs with small aspect ratios, their stress-strain responses can be approximately described by a bilinear law, similar to that for twisted and bent thick MWCNTs [16, 22].

The aspect-ratio dependent instability of the MWCNTs explains well two sets of experiments by Yap et al. [26] and Waters et al. [27, 28]. Since the MWCNTs used in the experiments of Yap et al. were of a relatively large aspect ratio, negative moduli were observed, just as for the 10-walled MWCNT studied here. In contrast, the MWCNTs used in the experiments of Waters et al. had small aspect ratios, i.e., they were very short, thick MWCNTs. The post-buckling mechanical response was more like that of the 25-walled MWCNT studied here. Such short, thick MWCNTs do not possess a negative modulus. We also found that, as the compressive strain increases further, the MWCNT will eventually bend, and the deformation morphology resembles the description of Waters et al. (i.e., highly distorted cross-sections and wrinkled side-walls). Since our model cannot address the activities of defects, particularly the nucleation and motion of the 5/7 dislocations in CNTs, it is incapable of reproducing the hysteresis observed in the experiments of Waters et al.

\section{Conclusions}

Our coarse-grained simulations predict, for the first time, unique evolving deformation morphologies in uniaxially compressed short, thick MWCNTs, including wave-like rippling, and symmetric and helical diamond patterns. The evolution of the post-buckling morphologies of MWCNTs depends on their aspect ratios, and is a result of coordinated deformations of the inner and outer shells. Our simulations show that onset buckling strain of MWCNTs with small aspect ratios under axial compression can be well predicted by the shell buckling theory, while that for SWCNTs with large aspect ratios can be predicted by Euler beam theory. Neither theory can describe the buckling of MWCNTs with moderate aspect ratios. Due to the stiffening effect of the inter-shell vdW interactions, the buckling strain of the outermost shells is delayed by a factor of $\alpha \approx 1.41$. The post-buckling modulus is substantially reduced, and increases monotonically with increasing thickness. The mechanical responses for very short, thick MWCNTs under uniaxial compression can be approximated by a bilinear law for a large range of the applied compressive strain. Our simulation results agree with, and explain, previous experimental data.

\section{Acknowledgements}

We gratefully acknowledge support from the National Science Foundation (NSF) grants under Awards Nos. 0826841 and 0600642 (Clark V. Cooper, program manager), and K. J. H. acknowledges NSF financial support under Grant Nos. CMMI 09-52565 and CMMI 09-06361.

\section{References}

[1] Baughman, R. H.; Zakhidov, A. A.; de Heer, W.A. Carbon nanotubes-The route toward applications. Science 2002, 297, 787-792.

[2] Zhang, S. L.; Liu, W. K.; Ruoff, R. S. Atomistic simulations of double-walled carbon nanotubes (DWCNTs) as rotational bearings. Nano Lett. 2004, 4, 293-297.

[3] Tombler, T. W.; Zhou, C. W.; Alexseyev, L.; Kong, J.; Dai, H. J.; Lei, L.; Jayanthi, C. S.; Tang, M. J.; Wu, S. Y. 
Reversible electromechanical characteristics of carbon nanotubes under local-probe manipulation. Nature 2000, 405, 769-772.

[4] Papadakis, S. J.; Hall, A. R.; Williams, P. A.; Vicci, L.; Falvo, M. R.; Superfine, R.; Washburn, S. Resonant oscillators with carbon-nanotube torsion springs. Phys. Rev. Lett. 2004, 93, 146101.

[5] Poncharal, P.; Wang, Z. L.; Ugarte, D.; de Heer, W. A. Electrostatic deflections and electromechanical resonances of carbon nanotubes. Science 1999, 283, 1513-1516.

[6] Cao, G. X.; Chen, X. Buckling behavior of single-walled carbon nanotubes and a targeted molecular mechanics approach. Phys. Rev. B 2006, 74, 165422.

[7] Sears, A.; Batra, R. C. Buckling of multiwalled carbon nanotubes under axial compression. Phys. Rev. B 2006, 73, 085410.

[8] Yakobson, B. I.; Brabec, C. J.; Bernholc, J. Nanomechanics of carbon tubes: Instabilities beyond linear response. Phys. Rev. Lett. 1996, 76, 2511-2514.

[9] Zhang, S. L.; Khare, R.; Belytschko, T.; Hsia, K. J.; Mielke, S. L.; Schatz, G. C. Transition states and minimum energy pathways for the collapse of carbon nanotubes. Phys. Rev. B 2006, 73, 075423.

[10] Khare, R.; Mielke, S. L.; Paci, J. T.; Zhang, S. L.; Ballarini, R.; Schatz, G. C.; Belytschko, T. Coupled quantum mechanical/molecular mechanical modeling of the fracture of defective carbon nanotubes and graphene sheets. Phys. Rev. B 2007, 75, 075412.

[11] Buehler, M. J.; Kong, Y.; Gao, H. J. Deformation mechanisms of very long single-wall carbon nanotubes subject to compressive loading. J. Eng. Mater-T. ASME 2004, 126, 245-249.

[12] Mahadevan, L.; Bico, J.; McKinley, G. Popliteal rippling of layered elastic tubes and scrolls. Europhys. Lett. 2004, 65, 323-329.

[13] Arias, I.; Arroyo, M. Size-dependent nonlinear elastic scaling of multiwalled carbon nanotubes. Phys. Rev. Lett. 2008, 100, 085503.

[14] Arroyo, M.; Arias, I. Rippling and a phase-transforming mesoscopic model for multiwalled carbon nanotubes. $J$. Mech. Phys. Solids 2008, 56, 1224-1244.

[15] Bower, C.; Rosen, R.; Jin, L.; Han, J.; Zhou, O. Deformation of carbon nanotubes in nanotube-polymer composites. Appl. Phys. Lett. 1999, 74, 3317-3319.

[16] Huang, X.; Zou, J.; Zhang, S. L. Bilinear responses and rippling morphologies of multiwalled carbon nanotubes under torsion. Appl. Phys. Lett. 2008, 93, 031915.
[17] Kuzumaki, T.; Hayashi, T.; Ichinose, H.; Miyazawa, K.; Ito, K.; Ishida, Y. In-situ observed deformation of carbon nanotubes. Philos. Mag. A 1998, 77, 1461-1469.

[18] Lourie, O.; Cox, D. M.; Wagner, H. D. Buckling and collapse of embedded carbon nanotubes. Phys. Rev. Lett. 1998, 81, 1638-1641.

[19] Pantano, A.; Boyce, M. C.; Parks, D. M. Nonlinear structural mechanics based modeling of carbon nanotube deformation. Phys. Rev. Lett. 2003, 91, 145504.

[20] Pantano, A.; Boyce, M. C.; Parks, D. M. Mechanics of axial compression of single and multi-wall carbon nanotubes. $J$. Eng. Mater-T. ASME 2004, 126, 279-284.

[21] Pantano, A.; Parks, D. M.; Boyce, M. C. Mechanics of deformation of single- and multi-wall carbon nanotubes. $J$. Mech. Phys. Solids 2004, 52, 789-821.

[22] Zou, J.; Huang, X.; Arroyo, M.; Zhang, S. L. Effective coarse-grained simulations of super-thick multi-walled carbon nanotubes under torsion. J. Appl. Phys. 2009, 105, 031915.

[23] Arroyo, M.; Belytschko, T. Nonlinear mechanical response and rippling of thick multiwalled carbon nanotubes. Phys. Rev. Lett. 2003, 91, 215505.

[24] Huang, Y.; Wu, J.; Hwang, K. C. Thickness of graphene and single-wall carbon nanotubes. Phys. Rev. B 2006, 74, 245413.

[25] Yap, H. W.; Lakes, R. S.; Carpick, R. W. Negative stiffness and enhanced damping of individual multiwalled carbon nanotubes. Phys. Rev. B 2008, 77, 045423.

[26] Yap, H. W.; Lakes, R. S.; Carpick, R. W. Mechanical instabilities of individual multiwalled carbon nanotubes under cyclic axial compression. Nano Lett. 2007, 7, 1149-1154.

[27] Waters, J. F.; Riester, L.; Jouzi, M.; Guduru, P. R.; Xu, J. M. Buckling instabilities in multiwalled carbon nanotubes under uniaxial compression. Appl. Phys. Lett. 2004, 85, 1787-1789.

[28] Waters, J. F.; Guduru, P. R.; Jouzi, M.; Xu, J. M.; Hanlon, T.; Suresh, S. Shell buckling of individual multiwalled carbon nanotubes using nanoindentation. Appl. Phys. Lett. 2005, 87, 103109.

[29] Jensen, K.; Mickelson, W.; Kis, A.; Zettl, A. Buckling and kinking force measurements on individual multiwalled carbon nanotubes. Phys. Rev. B 2007, 76, 195436.

[30] Dumitrica, T.; Hua, M.; Yakobson, B. I. Symmetry-, time-, and temperature-dependent strength of carbon nanotubes. Proc. Natl. Acad. Sci. USA 2006, 103, 6105-6109.

[31] Huang, J. Y.; Chen, S.; Wang, Z. Q.; Kempa, K.; Wang, Y. 
M.; Jo, S. H.; Chen, G.; Dresselhaus, M. S.; Ren, Z. F. Superplastic carbon nanotubes-Conditions have been discovered that allow extensive deformation of rigid single-walled nanotubes. Nature 2006, 439, 281.

[32] Mielke, S. L.; Troya, D.; Zhang, S.; Li, J. L.; Xiao, S. P.; Car, R.; Ruoff, R. S.; Schatz, G. C.; Belytschko, T. The role of vacancy defects and holes in the fracture of carbon nanotubes. Chem. Phys. Lett. 2004, 390, 413-420.

[33] Zhang, S.; Zhu, T. Atomic geometry and energetics of carbon nanotube necking. Philos. Mag. Lett. 2007, 87, 567-574.

[34] Zhang, S. L.; Mielke, S. L.; Khare, R.; Troya, D.; Ruoff, R. S.; Schatz, G. C.; Belytschko, T. Mechanics of defects in carbon nanotubes: Atomistic and multiscale simulations. Phys. Rev. B 2005, 71, 115403.

[35] Terdalkar, S. S.; Zhang, S. L.; Rencis, J. J.; Hsia, K. J. Molecular dynamics simulations of ion-irradiation induced deflection of 2D graphene films. Int. J. Solids Struct. 2008, 45, 3908-3917.

[36] Ru, C. Q. Degraded axial buckling strain of multiwalled carbon nanotubes due to interlayer slips. J. Appl. Phys. 2000, 89, 3326-3433.

[37] Brenner, D. W.; Shenderova, O. A.; Harrison, J. A.; Stuart, S. J.; Ni, B.; Sinnott, S. B. A second-generation reactive empirical bond order (REBO) potential energy expression for hydrocarbons. J. Phys.-Condens. Mat. 2002, 14, 783-802.
[38] Girifalco, L. A.; Hodak, M.; Lee, R. S. Carbon nanotubes, buckyballs, ropes, and a universal graphitic potential. Phys. Rev. B 2000, 62, 13104-13110.

[39] Arroyo, M.; Belytschko, T. An atomistic-based finite deformation membrane for single layer crystalline films. $J$. Mech. Phys. Solids 2002, 50, 1941-1977.

[40] Arroyo, M.; Belytschko, T. Finite element methods for the non-linear mechanics of crystalline sheets and nanotubes. Int. J. Numer. Meth. Eng. 2004, 59, 419-456.

[41] Zhang, S.; Khare, R.; Belytschko, T. A bridging domain and strain computation method for couple atomisticcontinuum modeling of solids. Int. J. Numer. Meth. Eng. 2007, 70, 913-933.

[42] Sinclair, J. E. Influence of interatomic force law and of kinks on propagation of brittle cracks. Philos. Mag. 1975, 31, 647-671.

[43] Kolmogorov, A. N.; Crespi, V. H. Smoothest bearings: Interlayer sliding in multiwalled carbon nanotubes. Phys. Rev. Lett. 2000, 85, 4727-4730.

[44] Kolmogorov, A. N.; Crespi, V. H. Registry-dependent interlayer potential for graphitic systems. Phys. Rev. B 2005, $71,235415$.

[45] Li, X. Y.; Yang, W.; Liu, B. Bending induced rippling and twisting of multiwalled carbon nanotubes. Phys. Rev. Lett. 2007, 98, 205502. 